\title{
A FAMILY OF SECOND DERIVATIVE SIMPSON'S TYPE BLOCK METHODS FOR STIFF SYSTEMS
}

\author{
Awari, Y.S. ${ }^{1 *}$, Shikaa S. ${ }^{2}$ and Stephen N.M. ${ }^{3}$ \\ ${ }^{* 1,2,3}$ Department of Mathematical Science, Taraba State University, Jalingo. Nigeria
}

\section{*Corresponding Author: -}

\begin{abstract}
: -
In this paper, we developed a new family of self-starting second derivative Simpson's type block methods (SDSM) of uniform order $p=2 k+2$ for step number $k \leq 6$. The new block methods for $k=2,3, \ldots, 6$ were seen to possess good stability property as they possessed good regions of absolute stability. They were also found to be consistent, zero stable and A-stable (Fig.4). This essential property made them suitable for the solution of stiff system of ordinary differential equations. Four numerical examples were considered and results obtained show improved accuracy in terms of their Maximum absolute errors when compared with the work of existing scholars. The newly developed block methods were seen to approximate well with the stiff Ode Solver (Fig. 5, 6, 7 and 8).
\end{abstract}

Keywords: Second derivative LMM, stiff ODE, block method, Simpson's method, stiffness ratio. 


\section{INTRODUCTION}

One major concern of the computational scientist is the numerical integration of stiff ordinary differential equations. Most real-life problems are modeled into system of ordinary differential equations, some of these equations exhibit behavior known as stiffness. Interest in stiff systems appeared initially in the $20^{\text {th }}$ century in radio engineering (e.g the Van der Pol problem). One of the first attempts to cope with the difficulties of stiffness was suggested by [8].

Recently, [30] introduced Second derivative Simpson's block method for $k=2$ for stiff ODEs of the form

$$
y^{\prime}(x)=f(x, y(x)), y\left(x_{0}\right)=y_{0}
$$

on the interval $I=\left[x_{0}, x_{N}\right]$, where $y:\left[x_{0}, x_{N}\right] \rightarrow R^{m}$ and $f:\left[x_{0}, x_{N}\right] \times R^{m} \rightarrow R^{m}$.

A potentially good numerical method for the solution of stiff systems of ODEs must have good accuracy and reasonable wide region of absolute stability [9].

The search for efficient, more accurate higher order A-stable block methods for the solution of stiff ODEs is now evolving $[3,4,8,14,15,18$ and 24]. It is for this reason that we developed more efficient methods that should address the problem of stiffness.

\section{Lemma 1.1}

Equation (1.1) is called stiff differential equation if its Jacobian (in the neighborhood of the solution) has eigenvalues that verify $\frac{\max \left|\operatorname{Re} \lambda_{i}\right|}{\min \left|\operatorname{Re} \lambda_{i}\right|}>>0$ that is if

(i) $\quad \operatorname{Re} \lambda_{i}<0, i=1,2, \ldots, m$ and

$$
\frac{\max _{i=1,2, \ldots, m}\left|\operatorname{Re} \lambda_{i}\right|}{\min _{i=1,2, \ldots, m}\left|\operatorname{Re} \lambda_{i}\right|}>0
$$

where $\lambda_{i}, i=1,2, \ldots, m$ are the eigenvalues of (1.1) (Lambert, 1973).

\section{Proof}

To prove lemma 1.1, we consider a system of first order ordinary differential equations

$$
\begin{aligned}
& y_{1}^{\prime}=-0.1 y_{1}-0.4 y_{2} \\
& y_{2}^{\prime}=-50 y_{2} \\
& y_{3}^{\prime}=70 y_{2}-120 y_{3}
\end{aligned}
$$

Then the Jacobian

$$
\begin{gathered}
J=\left(\begin{array}{lll}
\frac{\partial f_{1}}{\partial y_{1}} & \frac{\partial f_{1}}{\partial y_{2}} & \frac{\partial f_{1}}{\partial y_{3}} \\
\frac{\partial f_{2}}{\partial y_{1}} & \frac{\partial f_{2}}{\partial y_{2}} & \frac{\partial f_{2}}{\partial y_{3}} \\
\frac{\partial f_{3}}{\partial y_{1}} & \frac{\partial f_{3}}{\partial y_{2}} & \frac{\partial f_{3}}{\partial y_{3}}
\end{array}\right)=\left(\begin{array}{ccc}
-0.1 & -0.4 & 0 \\
0 & -50 & 0 \\
0 & 70 & -120
\end{array}\right) \\
\text { Now, } \left.|J \lambda-I|=\mid \begin{array}{ccc}
-0 . \lambda-1 & -0.4 \lambda & 0 \\
0 & -50 \lambda-1 & 0 \\
0 & 70 \lambda & -120 \lambda-1
\end{array}\right) \mid
\end{gathered}
$$

clearly, condition (i) and (ii) is satisfied with stiffness ratio $S=1.25 \times 10^{3}$

We extended the idea in [30] for $k=3,4, \ldots, 6$ and investigated their stability property; we also implemented the new block methods on some stiff ODEs occurring in real life.

The Conventional Second Derivative Linear Multistep Method (SDLMM) is written in the form

$$
\sum_{i=0}^{k} \phi_{i} y_{n+i}=h \sum_{i=0}^{k} \varphi_{i} f_{n+i}+h^{2} \sum_{i=0}^{k} \psi_{i} f_{n+i}^{\prime}
$$

where, $\phi_{i}, \varphi_{i}$ and $\psi_{i}$ are coefficients of the methods to be determined and $f_{n+i}^{\prime}=y_{n+i}^{\prime \prime}$.

A lot of scholars have considered the computational treatment for the solution of (1.1) through the second derivative 
method for instance [10, 11, 12, 18, 21, 25, 26 and 30].

Block methods were introduced to both improve the stability of methods and provide the $k-1$ Starting values to $k$ - step LMM. They are usually set of LMMs simultaneously applied to (1.1) and then

combined to yield better approximations. They have the capacity to generate simultaneously $k$ approximate solutions. Some of the scholars that have written extensively on block methods include but not limited to the following $[1,5,7,10$, 13, 17, 27, 28 and 29].

This paper is organized in the following manner. Section 2 will contain a discussion of the derivative of the methods with some few definitions, theorems and proofs. Section 3 contains the stability property of the family of second derivative Simpson's type block methods. In section 4, we shall present some numerical examples to showcase the efficiency and accuracy of the methods, while section 5 shall consists of discussion of results and conclusion.

\section{METHODOLOGY}

Our interest in this paper is to extend the idea in [30] to derive and implement a family of Second Derivative Simpson's type block Methods (SDSM) on (1.1) for $k=2,3, \ldots, 6$. This can be achieved through the derivation of a class of main methods and their associated additional methods.

In the spirit of [30], we constructed the main method for SSDSM in the form $y_{n} \square I y_{n} \square h_{\square} \square j \square 0 f_{n} \square j$

$$
y_{n+i}-y_{n}=h \sum_{j=0}^{k} \varphi_{j} f_{n+j}+h^{2} \sum_{j=0}^{k} \psi_{j} f_{n+j}^{\prime}, i=2,3, \ldots, 6
$$

where, $\varphi_{i}$ and $\psi_{i}$ are coefficients of the methods to be determine. We also have that

$$
\begin{aligned}
& y_{n+i}=y\left(x_{n+i}\right) \\
& f_{n+i}=f\left(x_{n+i}, y\left(x_{n+i}\right)\right) \\
& f_{n+i}^{\prime}=\frac{d f(x, y(x))}{d x}, i=0,1, \ldots, 6
\end{aligned}
$$

where, $y_{n}+i$ is an approximation to the theoretical solution $y\left(x_{n}+i\right)$.

Then the additional method can be constructed in the form

$$
y_{n+i-1}-y_{n}=h \sum_{j=0}^{k} \varphi_{j} f_{n+j}+h^{2} \sum_{j=0}^{k} \psi_{j} f_{n+j}^{\prime}, i=2,3, \ldots, 6
$$

\subsection{Specification of the methods}

In other to specify the methods (2) and (3), we seek a continuous interpolant of the SDSM to approximate the theoretical solution of (1.1). We assume that the solution of (1.1) is in the range $I=\left(x_{0}, x_{N}\right)$ by interpolating the function

$$
y(x)=h \sum_{i=0}^{2 k+2} \tau_{i} x^{i}
$$

where, ${ }^{\tau_{i}}$ are unknown coefficients of the method to be computed. The continuous SDSM is constructed by imposing the following conditions

$$
\sum_{i=0}^{k} \varphi_{i}=k \text {, that is, summation of the coefficients of } f_{n+j} \text { of the main method is equal to the step number } k \text {. }
$$

ii. The coefficients of $f_{n+i}^{\prime}, i=0,1, \ldots, k$ of the main method and those of the first characteristic polynomial are somewhat symmetric.

iii. $y\left(x_{n}\right)=y_{n}$

iv. $y^{\prime}\left(x_{n+i}\right)=f_{n+i}, i=0,1, \ldots, 6$

v. $y^{\prime \prime}\left(x_{n+i}\right)=f_{n+i}^{\prime}, i=0,1, \ldots, 6$

The conditions stated above are then used to solve for $\tau_{i}$. The continuous SDSM is derived by substituting the values of $\tau_{i}$ into equation (2.4). After some manipulations, the continuous approximation is expressed in the form 


$$
y(x)=y_{n}+h \sum_{j=0}^{k} \varphi_{j}(x) f_{n+j}+h^{2} \sum_{j=0}^{k} \psi_{j}(x) f_{n+j}^{\prime}
$$

Evaluating (2.5) at $x=x_{n+i}, i=1, \ldots, 6$, we obtained the desire set of main method and its associated final additional methods.

Accordingly, we presented the following set of Equations.

\section{Main Methods}

$$
\begin{aligned}
& \text { order } 2 k+2=6(S S D S M) \\
& y_{n+2}-y_{n}=\frac{h}{15}\left(7 f_{n+2}+16 f_{n+1}+7 f_{n}\right)-\frac{h^{2}}{15}\left(f_{n+2}^{\prime}-f_{n}^{\prime}\right) \\
& \text { order } 2 k+2=8(E S D S M) \\
& y_{n+3}-y_{n}=\frac{h}{224}\left(93 f_{n+3}+243 f_{n+2}+243 f_{n+1}+93 f_{n}\right)-\frac{h^{2}}{1120}\left(57 f_{n+3}^{\prime}-81 f_{n+2}^{\prime}+81 f_{n+1}^{\prime}-57 f_{n}^{\prime}\right) \\
& \text { order } 2 k+2=10(\text { TSDSM }) \\
& y_{n+4}-y_{n}=\frac{h}{8505}\left(3202 f_{n+4}+8192 f_{n+3}+11232 f_{n+2}+8192 f_{n+1}+3202 f_{n}\right)-\frac{h^{2}}{2835}\left(116 f_{n+4}^{\prime}\right. \\
& \left.-512 f_{n+3}^{\prime}+512 f_{n+1}^{\prime}-116 f_{n}^{\prime}\right) \\
& \text { order } 2 k+2=12\left(T^{*} S D S M\right) \\
& y_{n+5}-y_{n}=\frac{h}{912384}\left(319085 f_{n+5}+691875 f_{n+4}+1270000 f_{n+3}+1270000 f_{n+2}+691875 f_{n+1}\right. \\
& \left.+319085 f_{n}\right)-\frac{h^{2}}{1064448}\left(36975 f_{n+5}^{\prime}-314375 f_{n+4}^{\prime}-272500 f_{n+3}^{\prime}+272500 f_{n+2}^{\prime}+314375 f_{n+1}^{\prime}\right. \\
& \left.-36975 f_{n}^{\prime}\right)
\end{aligned}
$$

\section{Additional Methods}

$$
\begin{gathered}
y_{n+1}-y_{n}=\frac{h}{240}\left(11 f_{n+2}+128 f_{n+1}+101 f_{n}\right)-\frac{h^{2}}{240}\left(3 f_{n+2}^{\prime}+40 f_{n+1}^{\prime}-13 f_{n}^{\prime}\right) \\
\text { order } 2 k+2=8(\text { ESDSM }) \\
y_{n+2}-y_{n}=\frac{h}{567}\left(20 f_{n+3}+351 f_{n+2}+540 f_{n+1}+223 f_{n}\right)-\frac{h^{2}}{945}\left(8 f_{n+3}^{\prime}+171 f_{n+2}^{\prime}+144 f_{n+1}^{\prime}-43 f_{n}^{\prime}\right) \\
y_{n+1}-y_{n}=\frac{h}{18144}\left(397 f_{n+3}+2403 f_{n+2}+8451 f_{n+1}+6893 f_{n}\right)-\frac{h^{2}}{30240}\left(163 f_{n+3}^{\prime}+2421 f_{n+2}^{\prime}+\right. \\
\left.7659 f_{n+1}^{\prime}-1283 f_{n}^{\prime}\right) \quad \text { order } 2 k+2=10(T S D S M) \\
y_{n+3}-y_{n}=\frac{h}{17920}\left(411 f_{n+4}+11376 f_{n+3}+20736 f_{n+2}+14736 f_{n+1}+6501 f_{n}\right)-\frac{h^{2}}{8960}\left(45 f_{n+4}^{\prime}+\right. \\
\left.1464 f_{n+3}^{\prime}+2268 f_{n+2}^{\prime}+2232 f_{n+1}^{\prime}-339 f_{n}^{\prime}\right) \\
y_{n+2}-y_{n}=\frac{h}{68040}\left(1153 f_{n+4}+12608 f_{n+3}+44928 f_{n+2}+52928 f_{n+1}+24463 f_{n}\right)-\frac{h^{2}}{11340}\left(43 f_{n+4}^{\prime}\right. \\
\left.+992 f_{n+3}^{\prime}+4536 f_{n+2}^{\prime}+3040 f_{n+1}^{\prime}-421 f_{n}^{\prime}\right) \\
y_{n+1}-y_{n}=\frac{h}{4354560}\left(59681 f_{n+4}+613456 f_{n+3}+711936 f_{n+2}+1429936 f_{n+1}+1539551 f_{n}\right) \\
\frac{h^{2}}{725760}\left(2237 f_{n+4}^{\prime}+49720 f_{n+3}^{\prime}+183708 f_{n+2}^{\prime}+249656 f_{n+1}^{\prime}-26051 f_{n}^{\prime}\right) \\
\text { order } 2 k+2=12\left(T^{*} S D S M\right)
\end{gathered}
$$




$$
\begin{aligned}
& y_{n+4}-y_{n}=\frac{h}{22275}\left(336 f_{n+5}+13910 f_{n+4}+31360 f_{n+3}+22560 f_{n+2}+13360 f_{n+1}+7574 f_{n}\right) \\
& -\frac{h^{2}}{10395}\left(32 f_{n+5}^{\prime}+1492 f_{n+4}^{\prime}+3456 f_{n+3}^{\prime}+6400 f_{n+2}^{\prime}+3744 f_{n+1}^{\prime}-340 f_{n}^{\prime}\right) \\
& y_{n+3}-y_{n}=\frac{h}{1408000}\left(16617 f_{n+5}+284775 f_{n+4}+1316400 f_{n+3}+1316400 f_{n+2}+812775 f_{n+1}\right. \\
& \left.+477033 f_{n}\right)-\frac{h^{2}}{985600}\left(2421 f_{n+5}^{\prime}+79875 f_{n+4}^{\prime}+518340 f_{n+3}^{\prime}+664380 f_{n+2}^{\prime}+363645 f_{n+1}^{\prime}\right. \\
& \left.-31989 f_{n}^{\prime}\right) \\
& y_{n+2}-y_{n}=\frac{h}{891000}\left(9734 f_{n+5}+161325 f_{n+4}+407200 f_{n+3}+407200 f_{n+2}+495450 f_{n+1}\right. \\
& \left.+301091 f_{n}\right)-\frac{h^{2}}{415800}\left(948 f_{n+5}^{\prime}+30610 f_{n+4}^{\prime}+173840 f_{n+3}^{\prime}+325120 f_{n+2}^{\prime}+156500 f_{n+1}^{\prime}\right. \\
& \left.-13422 f_{n}^{\prime}\right) \\
& y_{n+1}-y_{n}=\frac{h}{22809600}\left(22134 f_{n+5}+3616235 f_{n+4}+8648560 f_{n+3}-362640 f_{n+2}+\right. \\
& \left.3053035 f_{n+1}+7633061 f_{n}\right)-\frac{h^{2}}{5322240}\left(10795 f_{n+5}^{\prime}+345053 f_{n+4}^{\prime}+1914300 f_{n+3}^{\prime}+\right. \\
& \left.3131972 f_{n+2}^{\prime}+2335779 f_{n+1}^{\prime}-168491 f_{n}^{\prime}\right) \\
& \text { order } 2 k+2=14 \text { (FSDSM) } \\
& y_{n+5}-y_{n}=\frac{h}{996323328}\left(10485255 f_{n+6}+609445680 f_{n+5}+1719564375 f_{n+4}+1617920000 f_{n+3}\right. \\
& \left.+379475625 f_{n+2}+322599120 f_{n+1}+3222126585 f_{n}\right)-\frac{h^{2}}{498161664}\left(1010975 g_{n+6}+64098000 g_{n+5}\right. \\
& \left.+210324375 g_{n+4}+623480000 g_{n+3}+614964375 g_{n+2}+235515600 g_{n+1}-14560225 g_{n}\right) \\
& y_{n+4}-y_{n}=\frac{h}{152026875}\left(1291400 f_{n+6}+31986624 f_{n+5}+198075750 f_{n+4}+233536000 f_{n+3}+\right. \\
& \left.47175000 f_{n+2}+46977600 f_{n+1}+49065126 f_{n}\right)-\frac{h^{2}}{30405375}\left(50720 g_{n+6}+2270592 g_{n+5}+\right. \\
& 20164500 g_{n+4}+42099200 g_{n+3}+38908800 g_{n+2}+14532480 g_{n+1}-885268 g_{n} \text { ) } \\
& y_{n+3}-y_{n}=\frac{h}{2562560000}\left(20942669 f_{n+6}+508254480 f_{n+5}+2168488125 f_{n+4}+2699264000 f_{n+3}\right. \\
& \left.+688759875 f_{n+2}+775497456 f_{n+1}+826473395 f_{n}\right)-\frac{h^{2}}{256256000}\left(411921 g_{n+6}+18189360 g_{n+5}\right. \\
& \left.+147584025 g_{n+4}+390561600 g_{n+3}+333689625 g_{n+2}+123030576 g_{n+1}-7450095 g_{n}\right) \\
& y_{n+2}-y_{n}=\frac{h}{2432430000}\left(19341201 f_{n+6}+466919808 f_{n+5}+1957353375 f_{n+4}+1387808000 f_{n+3}\right. \\
& \left.-457058625 f_{n+2}+706775424 f_{n+1}+783720817 f_{n}\right)-\frac{h^{2}}{243243000}\left(380629 g_{n+6}+16742592 g_{n+5}\right. \\
& \left.+134615475 g_{n+4}+336793600 g_{n+3}+337970925 g_{n+2}+117681984 g_{n+1}-7057013 g_{n}\right) \\
& y_{n+1}-y_{n}=\frac{h}{622702080000}\left(4592987927 f_{n+6}+110327270448 f_{n+5}+457138998375 f_{n+4}\right. \\
& +300642304000 f_{n+3}-380416470375 f_{n+2}-68951829552 f_{n+1}+199368819177 f_{n} \text { ) } \\
& -\frac{h^{2}}{62270208000}\left(90441763 g_{n+6}+3963034512 g_{n+5}+31646886075 g_{n+4}+77935000000 g_{n+3}\right. \\
& +71514207675 g_{n+2}+33488665488 g_{n+1}-1784098013 g_{n} \text { ) }
\end{aligned}
$$




\section{ANALYSIS}

Dahlquist (1963) investigated the stability problem associated with stiff eqautions, the scholar introduced the concept of A-stability, he then outline the following definitions:

3.1 Order of accuracy and local truncation error: Following [13, 19 and 20], the local truncation error associated with our block methods is the linear difference operator

$$
L[y(x): h]=\sum_{j=0}^{k}\left\{\alpha_{j} y(x+j h)-h \varphi_{j} y^{\prime}(x+j h)-h^{2} \psi_{j} y^{\prime \prime}(x+j h)\right\}
$$

We assume that $y(x)$ is sufficiently differentiable and so the terms of (3.1) can be expanded in Taylor series about $x$ to give the expression

$$
L[y(x): h]=c_{0} z(x)+c_{1} h z^{\prime}(x)+c_{2} h^{2} z^{\prime \prime}(x)+\ldots+c_{q} h^{q} z^{(q)}(x)+\ldots
$$

where,

$$
\begin{aligned}
& c_{0}=\sum_{j=0}^{k} \alpha_{j} \\
& c_{1}=\sum_{j=1}^{k} j \alpha_{j}-\sum_{j=0}^{k} \varphi_{j} \\
& c_{2}=\frac{1}{2 !} \sum_{j=1}^{k} j^{2} \alpha_{j}-\sum_{j=1}^{k} j \varphi_{j}-\sum_{j=0}^{k} \psi_{j} \\
& \cdot \\
& c_{q}=\frac{1}{q !} \sum_{j=1}^{k} j^{q} \alpha_{j}-\frac{1}{(q-1) !} \sum_{j=1}^{k} j^{q-1} \varphi_{j}-\frac{1}{(q-2) !} \sum_{j=0}^{k} j^{q-2} \psi_{j}, q=3,4, \ldots
\end{aligned}
$$

The computation above leads to definition (3.1)

Definition 3.1: A numerical method is said to be of order $p$ if $c_{0}=c_{1}=c_{2}=\ldots=c_{p}$ is called the error constant. The local truncation error (LTE) of the method given by

\begin{tabular}{|c|c|c|c|}
\hline & & \multicolumn{2}{|c|}{ Error Constants } \\
\hline Method & order $p=2 k+2$ & Main Method & Additional Method $(s)$ \\
\hline SSDSM & 6 & $c_{7}=2.116 \times 10^{-4}$ & $c_{7}=1.058 \times 10^{-4}$ \\
\hline ESDSM & 8 & $c_{9}=2.870 \times 10^{-5}$ & $\begin{array}{l}c_{9}=1.638 \times 10^{-5} \\
c_{9}=1.232 \times 10^{-5}\end{array}$ \\
\hline TSDSM & 10 & $c_{11}=4.072 \times 10^{-6}$ & $\begin{array}{l}c_{11}=2.319 \times 10^{-6} \\
c_{11}=2.036 \times 10^{-6} \\
c_{11}=1.753 \times 10^{-6}\end{array}$ \\
\hline $\mathrm{T} * \mathrm{SDSM}$ & 12 & $c_{13}=6.275 \times 10^{-7}$ & $\begin{array}{l}c_{13}=3.471 \times 10^{-7} \\
c_{13}=3.204 \times 10^{-7} \\
c_{13}=3.072 \times 10^{-7} \\
c_{13}=2.804 \times 10^{-7}\end{array}$ \\
\hline FSDSM & 14 & $c_{15}=1.048 \times 10^{-7}$ & $\begin{array}{l}c_{15}=5.633 \times 10^{-8} \\
c_{15}=5.329 \times 10^{-8} \\
c_{15}=5.240 \times 10^{-8} \\
c_{15}=5.151 \times 10^{-8} \\
c_{15}=4.846 \times 10^{-8}\end{array}$ \\
\hline
\end{tabular}

$$
t_{n+k}=\vec{c}_{p+1} h^{(p+1)} y^{(p+1)} x_{n}+o\left(h^{(p+2)}\right)
$$

Following [16] and as a consequence of (3.1)-(3.3), we presents the order and error constants of the newly derived block methods in the table 1 .

Table 1: Order and error constants of the Second Derivative Simpson's Type Block Methods 


\subsection{Stability Analysis}

We consider the TSDSM as an example for computation of stability analysis, we obtained its zero stabilty and region of absolute stabilty.

The TSDSM can be represented by a matrix finite difference equation in block form as

$$
A^{(0)} Y_{\mu}=A^{(1)} Y_{\mu-1}+h\left[\varphi^{(0)} F_{\mu}+\varphi^{(1)} F_{\mu-1}\right]+h^{2}\left[\psi^{(0)} F_{\mu}^{\prime}+\psi^{(1)} F_{\mu-1}^{\prime}\right]
$$

where,

$$
\begin{aligned}
& Y_{\mu}=\left(y_{n+1}, \ldots, y_{n+4}\right)^{T}, Y_{\mu-1}=\left(y_{n-3}, \ldots, y_{n}\right)^{T}, F_{\mu}=\left(f_{n+1}, \ldots, f_{n+4}\right)^{T}, F_{\mu-1}=\left(f_{n-3}, \ldots, f_{n}\right)^{T}, \\
& F_{\mu}^{\prime}=\left(f_{n+1}^{\prime}, \ldots, f_{n+4}^{\prime}\right)^{T}, \quad F_{\mu-1}^{\prime}=\left(f_{n-3}^{\prime}, \ldots, f_{n}^{\prime}\right)^{T}
\end{aligned}
$$

the matrices $A^{(0)}, A^{(1)}, \varphi^{(0)}, \varphi^{(1)}, \psi^{(0)}$ and $\psi^{(1)}$ are matrices of dimension 4 defined as follows: $A^{(0)}$ is an identity matrix of dimension 4 .

$$
\begin{aligned}
& A^{(0)}=\left(\begin{array}{llll}
1 & 0 & 0 & 0 \\
0 & 1 & 0 & 0 \\
0 & 0 & 1 & 0 \\
0 & 0 & 0 & 1
\end{array}\right), A^{(1)}=\left(\begin{array}{llll}
0 & 0 & 0 & 1 \\
0 & 0 & 0 & 1 \\
0 & 0 & 0 & 1 \\
0 & 0 & 0 & 1
\end{array}\right), \\
& \varphi^{(0)}=\left(\begin{array}{cccc}
\frac{89371}{272160} & \frac{103}{630} & \frac{38341}{272160} & \frac{59681}{4354560} \\
\frac{6616}{8505} & \frac{208}{315} & \frac{1576}{8505} & \frac{1153}{68040} \\
\frac{921}{1120} & \frac{81}{70} & \frac{711}{1120} & \frac{411}{17920} \\
\frac{8192}{8505} & \frac{416}{315} & \frac{8192}{8505} & \frac{3202}{8505}
\end{array}\right), \varphi^{(1)}=\left(\begin{array}{cccc}
0 & 0 & 0 & \frac{1539551}{4354560} \\
0 & 0 & 0 & \frac{24463}{68040} \\
0 & 0 & 0 & \frac{6501}{17920} \\
0 & 0 & 0 & \frac{3202}{8505}
\end{array}\right), \\
& \psi^{(0)}=\left(\begin{array}{cccc}
-\frac{31207}{90720} & -\frac{81}{320} & -\frac{1243}{18144} & -\frac{2237}{725760} \\
-\frac{152}{567} & -\frac{2}{5} & -\frac{248}{2835} & -\frac{43}{11340} \\
-\frac{279}{1120} & -\frac{81}{320} & -\frac{183}{1120} & -\frac{9}{1792} \\
-\frac{512}{2835} & 0 & \frac{512}{2835} & -\frac{116}{2835}
\end{array}\right), \quad \psi^{(1)}=\left(\begin{array}{cccc}
0 & 0 & 0 & \frac{26051}{725760} \\
0 & 0 & 0 & \frac{421}{11340} \\
0 & 0 & 0 & \frac{339}{8960} \\
0 & 0 & 0 & \frac{116}{2835}
\end{array}\right)
\end{aligned}
$$

Definition 3.2 [Chu and Hamilton 1987]: A block method is Zero-stable if the roots $R_{i, j}=1[1] k$

are of the first characteristic polynomial $\rho(R)=\operatorname{det}\left[\sum_{i=0}^{k} A_{j} R^{k-i}\right]=0, A_{0}=-I, \underset{\text { satisfies }}{ }\left|R_{j}\right| \leq 1$. one of the roots is +1 , we say this root is the principal root of $\rho(R)$.

Following definition (3.2), zero stability of the block method ( TSDSM) is concerned with the stability of the difference system in the limit as $h$ tends to zero. Subsequently, equation (3.4) tends to

$$
A^{(0)} Y_{\mu}=A^{(1)} Y_{\mu-1}
$$

whose first characteristics polynomial ${ }^{\rho(R)}$ given by

$$
\rho(R)=\operatorname{det}\left(R A^{(1)}-A^{(0)}\right)=R^{3}(R-1)
$$


Hence $R=0,0,0,1$. Following [13], the TSDSM is zero stable for ${ }^{\rho(R)}=0$ and satisfies $\left|R_{j}\right| \leq 1, j=1,2, \ldots, 4$ and for the root with $\left|R_{j}\right|=1$, the multiplicity does not exceed 1 .

Definition 3.3 [Dahlquist 1963, Fatunla 1991]: A numerical method is said to be A-stable if its region of absolute stability contains the whole of the laft hand complex half plane $\operatorname{Re}(\lambda h)<0$

The linear stability properties of TSDSM is discussed in the spirit of [15] and determined by expressing it in the form of (3.4) and apply the test problem

$$
y^{\prime}=\lambda y, y^{\prime \prime}=\lambda^{2} y, \lambda<0
$$

To yield

$$
Y_{\mu}=Q(z) Y_{\mu-1}, \quad z=\lambda h
$$

where

$$
Y_{\mu}=\left(\begin{array}{cccc}
1-\frac{89371}{272160} z+\frac{31207}{90720} z^{2} & \frac{81}{320} z^{2}-\frac{103}{630} z & \frac{1243}{18144} z^{2}-\frac{38341}{272160} z & \frac{2237}{725760} z^{2}-\frac{59681}{4354560} z \\
\frac{152}{567} z^{2}-\frac{6616}{8505} z & 1-\frac{208}{315} z+\frac{2}{5} z^{2} & \frac{248}{2835} z^{2}-\frac{1576}{8505} z & \frac{43}{11340} z^{2}-\frac{1153}{68040} z \\
\frac{279}{1120} z^{2}-\frac{921}{1120} z & \frac{81}{320} z^{2}-\frac{81}{70} z & 1-\frac{711}{1120} z+\frac{183}{1120} z^{2} & \frac{9}{1792} z^{2}-\frac{411}{17920} z \\
\frac{512}{2835} z^{2}-\frac{8192}{8505} z & -\frac{416}{315} z & -\frac{8192}{8505} z-\frac{512}{2835} z^{2} & 1-\frac{3202}{8505} z+\frac{116}{2835} z^{2}
\end{array}\right)
$$

the matrix $Q(z)$ is also given by

$$
Q(z)=\left(A^{(1)}-z \varphi^{(1)}-z^{2} \psi^{(1)}\right)^{-1} \cdot\left(A^{(0)}-z \varphi^{(0)}\right)
$$

The matrix $Q(z)$ has eigenvalues $\left\{\xi_{1}, \xi_{2}, \ldots, \xi_{k}\right\}=\{0,0, \ldots, 1\}$, where the dominant eigenvalues $\xi_{k}$ is the stability function $\xi(z)$ : which is a rational function with real coefficients given by

$$
\xi_{k}(z)=\frac{63\left(24 z^{8}+30 z^{7}+2090 z^{6}+9950 z^{5}+34095 z^{4}+84000 z^{3}+142800 z^{2}+151200 z+75600\right)}{1512 z^{8}-31230 z^{7}+115230 z^{6}-719325 z^{5}+176385 z^{4}-4935800 z^{3}+8119600 z^{2}-9525600 z+4762800}
$$

Thus, the absolute stability region of (3.10) is shown below: 


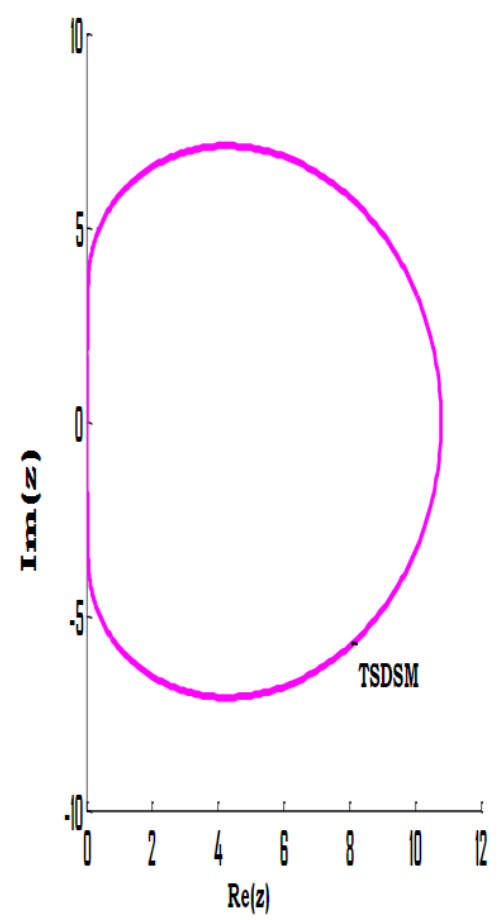

Fig.1: Stability Region of the TSDSM Block method.

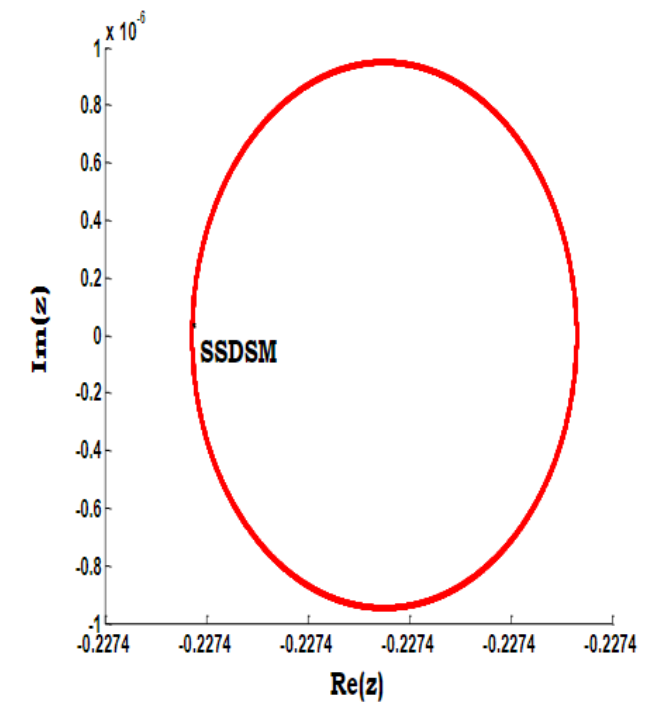

Fig.2: Stability Region of the discrete SSDSM (Main Method).

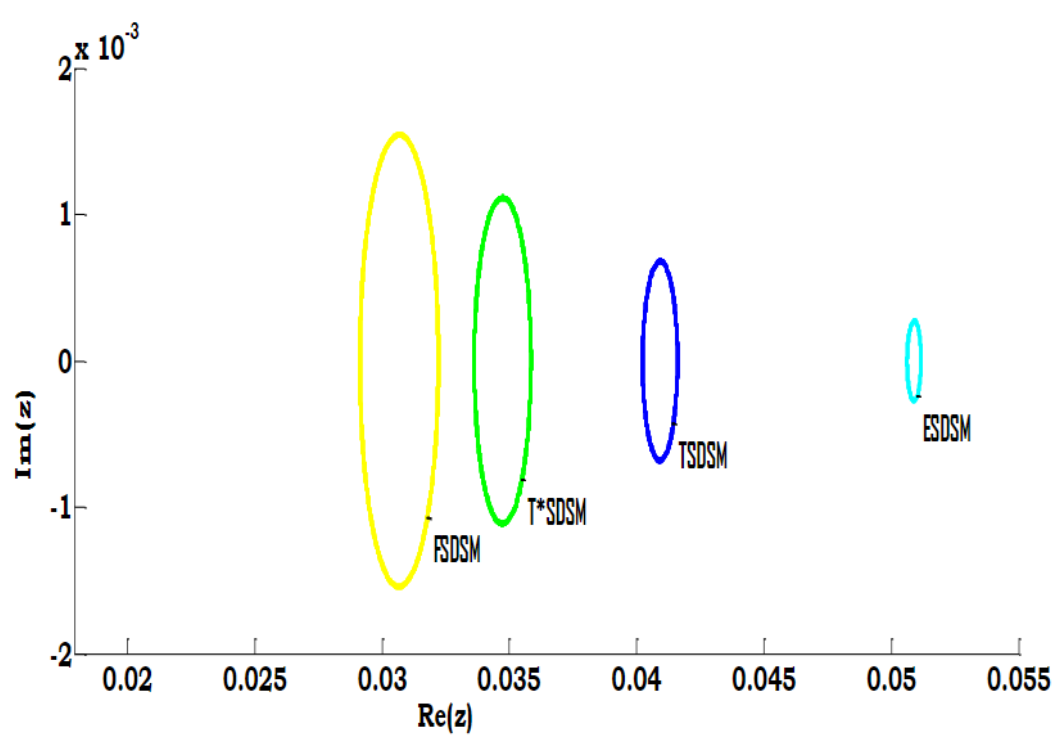

Fig.3: Stability Region of the discrete ESDSM, TSDSM, T*SDSM and FSDSM (Main Method) 


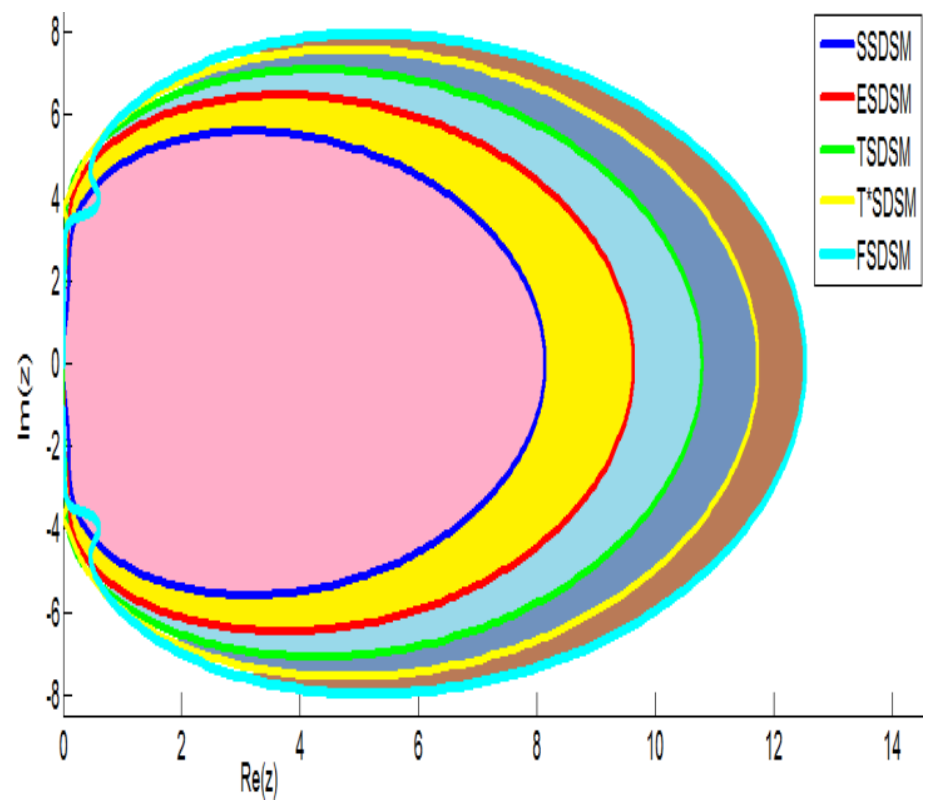

Fig.4: Stability Region of the SSDSM, ESDSM, TSDSM, T*SDSM and FSDSM (Block Method)

\section{NUMERICAL EXPERIMENTS}

In this section, the newly derived block methods were implemented on four stiff systems of ordinary differential equations occurring in real life. All computations were carried out using the MatLab code in MatLab 7.5.0 (R2007b) and Maple 18. The problems considered were in the form

$$
y_{i}^{\prime}=A y_{i}+\varphi(x), y_{i}(0)=\eta, i=1,2, \ldots, n
$$

Problem 1: Consider a Linear stiff system in 3 dimensions as in (4.1) where,

$$
A=\left(\begin{array}{ccc}
-21 & 19 & -20 \\
19 & -21 & 20 \\
40 & -40 & -40
\end{array}\right), \quad \eta=\left(\begin{array}{c}
1 \\
0 \\
-1
\end{array}\right), \quad \varphi(x)=\left(\begin{array}{l}
0 \\
0 \\
0
\end{array}\right)
$$

with analytical solution given by

$$
\left.\begin{array}{c}
\mathrm{y}_{1}=\frac{1}{2}\left(e^{-2 x}+e^{-40 x}(\cos (40 x)+\sin (40 x))\right) \\
\mathrm{y}_{2}=\frac{1}{2}\left(e^{-2 x}-e^{-40 x}(\cos (40 x)+\sin (40 x))\right) \\
\left.\mathrm{y}_{3}=-e^{-40 x}(\cos (40 x)+\sin (40 x))\right)
\end{array}\right\}
$$

Equation (4.3) was transformed into its second derivative as:

$$
\begin{array}{r}
V=\left(\begin{array}{ccc}
2 & 2 & 1600 \\
2 & 2 & -1600 \\
3200 & -3200 & 0
\end{array}\right) \\
y_{i}^{\prime \prime}=V y_{i}
\end{array}
$$

The SSDSM was applied to problem 4.1 where the maximum absolute errors in the interval $0<x<1$ were compared with methods derived by other scholars (see Table 2).

Table 2: Comparison of the newly block methods with Existing Methods for problem 4.1

\begin{tabular}{|l|l|l|l|l|c|c|c|c|}
\hline \multicolumn{1}{|c|}{$h=\frac{n \times 10}{100}$} & Steps & $\begin{array}{l}\text { SSDSM } \\
\text { Order 6 }\end{array}$ & $h=\frac{1}{2^{n} \times 100}$ & Steps & $\begin{array}{l}\text { SDGBDF4 } \\
\text { Order 4 [25] }\end{array}$ & $\begin{array}{l}\text { EH-OK5[10 ] } \\
\text { Order 5 }\end{array}$ & $\begin{array}{l}\text { GBDF8[6] } \\
\text { Order 8 }\end{array}$ & $\begin{array}{l}\text { ATBM7[2] } \\
\text { Order 7 }\end{array}$ \\
\hline$n=1$ & 0.1 & $9.86 \mathrm{e}-10$ & $n=0$ & 0.01 & $2.28 \mathrm{e}-17$ & $3.21 \mathrm{e}-13$ & $1.19 \mathrm{e}-3$ & $3.95 \mathrm{e}-6$ \\
\hline$n=2$ & 0.2 & $3.56 \mathrm{e}-15$ & $n=1$ & 0.005 & $1.56 \mathrm{e}-16$ & $1.01 \mathrm{e}-14$ & $1.30 \mathrm{e}-5$ & $2.91 \mathrm{e}-8$ \\
\hline$n=3$ & 0.3 & $3.92 \mathrm{e}-19$ & $n=2$ & 0.0025 & $1.02 \mathrm{e}-19$ & $3.18 \mathrm{e}-16$ & $1.08 \mathrm{e}-7$ & $2.21 \mathrm{e}-10$ \\
\hline$n=4$ & 0.4 & $5.68 \mathrm{e}-23$ & $n=3$ & 0.00125 & $6.21 \mathrm{e}-21$ & $9.96 \mathrm{e}-18$ & $1.08 \mathrm{e}-9$ & $6.65 \mathrm{e}-13$ \\
\hline$n=5$ & 0.5 & $1.04 \mathrm{e}-26$ & $n=4$ & 0.000625 & $9.45 \mathrm{e}-23$ & $3.11 \mathrm{e}-19$ & $9.41 \mathrm{e}-12$ & $2.69 \mathrm{e}-15$ \\
\hline
\end{tabular}




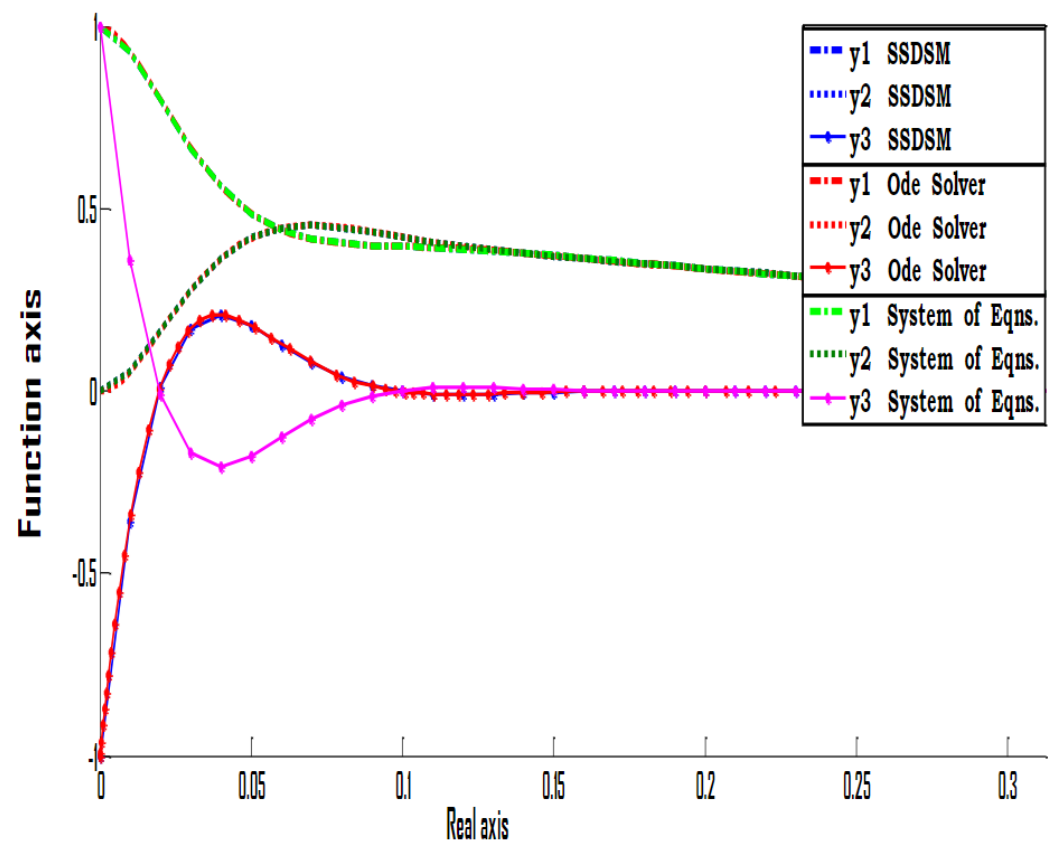

Fig. 5: Graphical Solution of problem 1 with SSDSM, $h=0.01$

Problem 2: We consider another stiff initial value problem in the form (4.1) given by

$$
A=\left(\begin{array}{ccc}
-0.1 & -0.4 & 0 \\
0 & -50 & 0 \\
0 & 70 & -120
\end{array}\right), \eta=\left(\begin{array}{l}
0 \\
1 \\
1
\end{array}\right), \varphi(x)=\left(\begin{array}{l}
0 \\
0 \\
0
\end{array}\right)
$$

we solve the problem at $x=2$ with $h=0.001$. Transforming (4.6) into second derivative resulted to:

$$
V=\left(\begin{array}{ccc}
0.01 & 20.04 & 0 \\
0 & -2500 & 0 \\
0 & -11900 & 14400
\end{array}\right)
$$

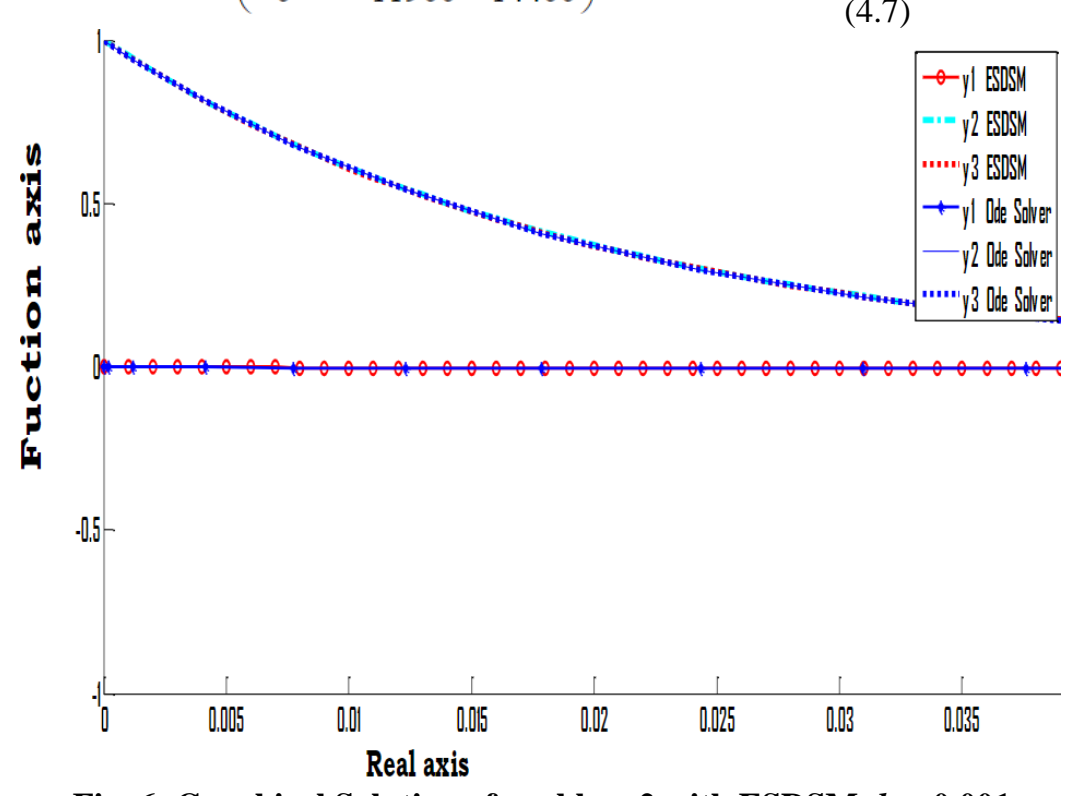

Fig. 6: Graphical Solution of problem 2 with $\operatorname{ESDSM,~} h=0.001$

Problem 3: A numerical example solved by [21].

$$
\begin{aligned}
& y_{1}^{\prime}=-\alpha y_{1}-\beta y_{2}+(\alpha+\beta-1) e^{-x} \\
& y_{2}^{\prime}=\beta y_{1}-\alpha y_{2}-(\alpha-\beta-1) e^{-x}
\end{aligned}
$$


with initial value $y(0)=(1,1)^{T}$. In order to make this system homogeneous, we introduce an additional variable $y_{3}^{\prime}=1, y_{3}(0)=0$. the eigenvalues of the Jacobian associated with the resulting system are $-\alpha \pm i \beta, 0$. this problem has theoretical solution as $y_{1}(x)=y_{2}(x)=e^{-x}$. Results are obtained when $\alpha=1, \beta=30$ and the value of $h$ chosen was 0.09

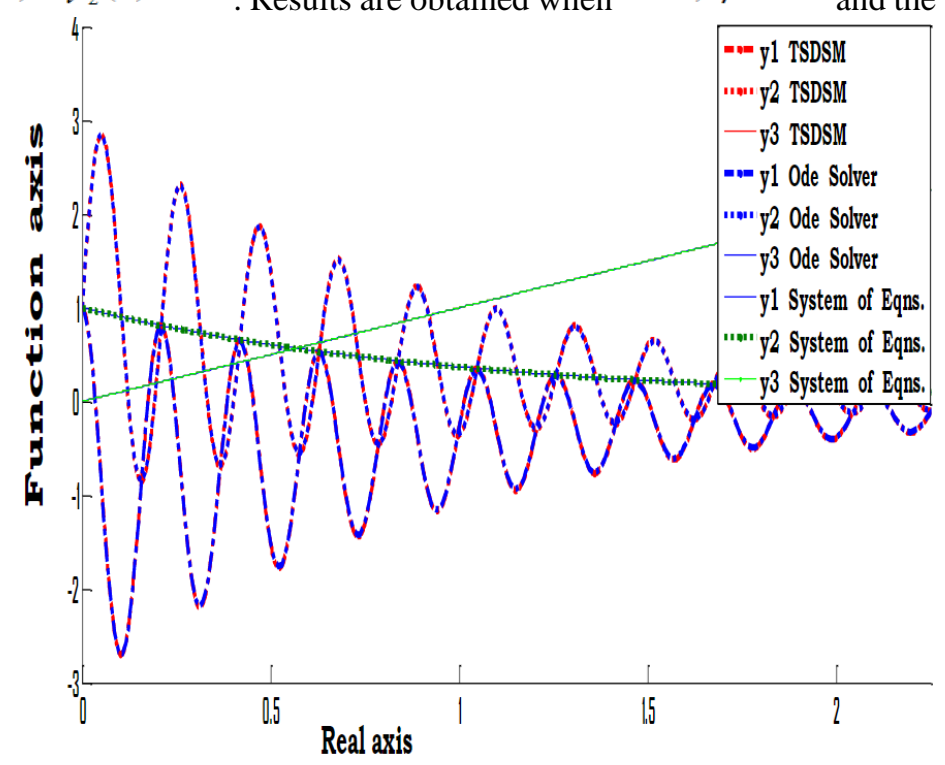

Fig. 7: Graphical Solution of problem 4.3 with TSDSM, $h=0.01$

Problem 4: To test the efficiency of the proposed algorithm we used the following stiff initial value problem arising from the biochemistry see [23].

$$
\begin{aligned}
& \frac{d y_{1}(t)}{d t}=\frac{1}{\alpha}\left(y_{1}(t)+y_{2}(t)-y_{1}(t) y_{2}(t)-q y_{1}^{2}(t)\right), \\
& \frac{d y_{2}(t)}{d t}=2 m y_{3}(t)-y_{2}(t)-y_{1}(t) y_{2}(t), \\
& \frac{d y_{3}(t)}{d t}=\frac{1}{r}\left(y_{1}(t)-y_{3}(t)\right), y_{1}(0)=a, y_{2}(0)=b, y_{3}(0)=d
\end{aligned}
$$

Here $\alpha, m, q$ and $r$ are some parameters, $a, b$ and $d$ are the initial values. For some values of parameters this model has a periodic solution very sensitive for the parameter values. Let the parameter values be as follows: $\alpha:=0.1 ; q:=0.01 ; m:=0.5 ; r:=1$ and the initial conditions are $a=0, b=0.5$ and $d=0.8$. The test problem was solved on the interval [0,30]. Problem 4 was extracted from the work of [22].

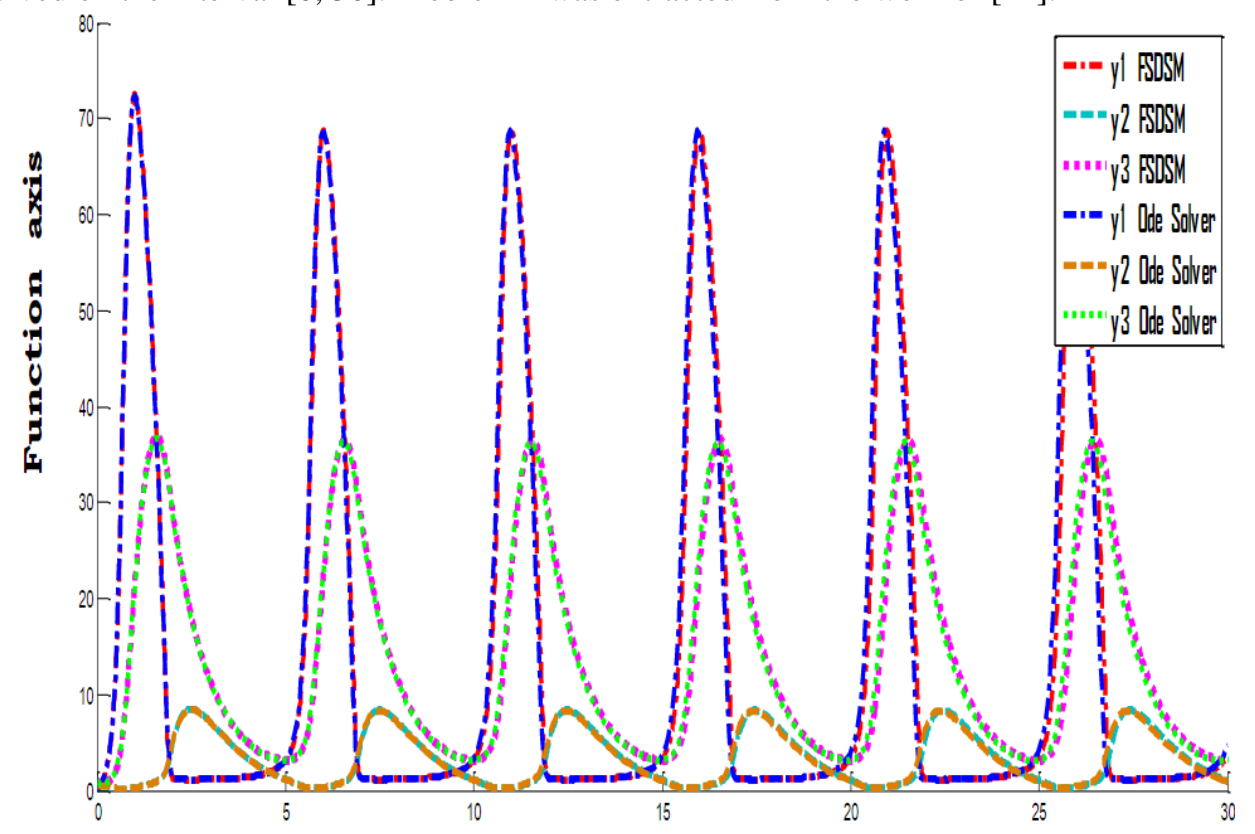

Fig. 8: Graphical Solution of problem 4.4 with FSDSM, $h=0.01$ 


\section{DISCUSSION OF RESULTS}

In this paper, we described the construction of a class of Second Derivative Simpson's type Block Methods (SDSM) of order $6,8,10,12$ and 14. These block methods were all found to be A-stable with order $p=2 k+2$ for step numbers $k=$ 2(3)6 which are appropriate for the numerical solution of system of stiff differential equations (Fig. 4). The discrete forms of the methods were found to have small absolute stability regions for the step numbers under consideration (Fig.2 and Fig. 3). The numerical results obtained were generated using codes written in MATLAB 7.5.0 (R2007b) and Maple 18. It is worth noting that the newly derived block methods approximated well with the Ode Solver (Ode 23s) (Fig. 5, 6 7 and 8) for the step numbers $k=2(3) 6$. Example, in Fig. 6, both the Ode Solver and ESDSM overlapped at y1, y2 and y3. Comparison of the new block methods with existing methods showed that the new methods perform much better in terms of accuracy. For instance, SSDSM of order 6 performs better in accuracy when compared with ATBM and GBDF8 of order 7 and 8 respectively (Table 2).

\section{CONCLUSION}

The Second Derivative Simpson's Type Block Methods (SDSM) was introduced in section (2). The newly developed class of methods was found to be all A-stable for values of $k=2(3) 6$ with uniform order $p=2 k+2$ (see Table 1 ) in section (3). They also possessed good stability regions suitable for the solution of stiff system of ODEs. Application of these methods to real life problems was carried out in section (4) and indicated that they were both accurate and very efficient, as a result of this therefore; the authors were of the opinion that they be employed for the solution of large stiff systems and possibly for solution of PDEs through the method of lines (MOL).

\section{Appendix}

SSDSM : Sixth order Second Derivative Simpson's Type Block Method ESDSM : Eighth order Second Derivative Simpson's Type Block Method TSDSM : Tenth order Second Derivative Simpson's Type Block Method

$T^{*} S D S M$ : Twelfth order Second Derivative Simpson's Type Block Method

FSDSM : Fourteenth order Second Derivative Simpson's Type Block Method

\section{Reference}

[1].Adeyeye Oluwaseun and Omar Zurni (2017): 4-Step 5-Point Hybrid Block Method for the direct Solution of Second Order Initial Value Problems. Journal of Mathematics and Computer Science, 17: 527-534

[2].Akinfenwa, O. A. (2011): Seven step Adams Type Block Method with Continuous Coefficient for Periodic Ordinary Differential Equation, Word Academy of Science, Engineering and Technology, 74: 848-853.

[3].Akinfenwa, O.A., et al (2017): Continuous L-Stable Multiderivative Hybrid Implicit Runge-Kutta Method of Order Five for the Integration of Stiff Problems, International Journal of Management and Applied Science, Vol. 3, Issue 1: $140-145$

[4].Ali, K. Ezzeddine and Gholamreza Hojjati (2011): Hybrid Extended Backward Differentiation Formulas for Stiff Systems. International Journal of Nonlinear Science, Vol. 12, No.2: 196-204

[5].Biala, T.A., and Jator, S.N. (2017): A Family of Boundary Value Methods for System of Second Order Boundary Value Problems. Hindawi International Journal of Differential Equations, Article ID: 2464759, 12 pages.

[6].Brugnano, L. and Trigiante, D. (2001): Block Implicit methods for ODEs in: D. Trigiante (Ed.), Recent trends in Numerical Analysis, New York: Nova Science Publ. inc.

[7].Chu, M.T. and Hamilton, H. (1987): Parallel solution of ODEs by multi-block methods, SIAM J. Sci. Stat. Comput.8:342-353

[8].Curtis, C. F. and Hirschfelder, J. O. (1952): Integration of Stiff Equations, National Academy of Sciences, 38,235 243.

[9].Dahlquist, G. (1963): A Special Stability Problem for Linear Multistep Methods, BIT. 3: 27-43.

[10]. Ehigie, J.O., and Okunuga, S.A. (2014): L( $\square$ )-Stable Second Derivative Block Multistep Formula for Stiff Initial Value Problems. IAENG International Journal of Applied Mathematics, 44(3): 157-162

[11]. Enright, W.H. (1974): Second Derivative Multistep Methods for Stiff Ordinary Differential Equations, SIAM, J. Num. Anal., 11:321-331.

[12]. Esuabana, I.M. and Ekoro, S.E. (2018): Derivation and Implementation of new Family of Second Derivative Hybrid Linear Multistep Methods for Stiff Ordinary Differential Equations. Global Journal of Mathematics, Vol.12, No.2: 821-828

[13]. Fatunla,S.O. (1991). Block methods for second order IVP's.,Inter.J.Comp.Maths.41: 55-63.

[14]. Grace, O. Akinlabi et al (2017): Hybrid Boundary Value Methods for the Solution of First Order Stiff Systems. International Journal of Circuits, Systems and Signal Processing, 11: 332-337

[15]. Hairer, E. and Wanner, G. (1996). Solving Ordinary Differential Equations II: Stiff and Differential-Algebraic Problems. Springer-Verlag, Second revised edition.

[16]. Henrici,P. (1962). Discrete variable methods for ODE's.John Wiley,New York.

[17]. Hijazi, M., and Abdelrahim, R. (2017): The Numerical Computation of Three Step Hybrid Block Method for Directly Solving Third Order Ordinary Differential Equations. Global Journal of Pure and Applied Mathematics, Vol. 13, No. 1: 89-103

[18]. Kumleng G.M., and Sirisena, U.W.W (2014): A( $\square$ )-Stable Order Ten Second Derivative Block Multistep Method for Stiff Initial Value Problems. International Journal of Mathematics and Statistics Invention (IJMSI), Vol. 2, Issue 10: $37-43$ 
[19]. Lambert,J.D. (1973). Computational methods for ordinary differential equations, John Wiley,New York.

[20]. Lambert,J.D. (1991). Numerical methods for ordinarydifferential systems.John Wiley,New York.

[21]. Mehdizadeh, K.M., Nasehi, O.N. and Hojjati, G. A. (2012): Class of second derivative multistep methods for stiff systems. Acta Universitatis Apulensis, No. 30: 171-188.

[22]. Miletics, E. and Moln'arka, G. Taylor Series Method with Numerical Derivatives for Numerical Solution of ODE Initial Value Problems, HU ISSN 1418-7108: HEJ Manuscript no.: ANM-030110-B, 2009.

[23]. Murray, J.B. (1977). Lectures on nonlinear-differential-equation models in biology, Clarendon Press, Oxford.

[24]. Mustafa, A. and Ibrahim, M.O. (2015): Solving systems of Stiff Ordinary Differential Equations from Model of Biochemical reaction Networks using Implicit Runge-Kutta Methods. International Journal of Engineering Sciences and Research Technology, 4(6): 348-356

[25]. Nwachukwu, G.C., and Okor, T. (2018): Second Derivative Generalized Backward Differentiation Formulae for Solving Stiff Problems. IAENG International Journal of Applied Mathematics, 48(1).

[26]. Okuonghae, R.I., and Ikhile, M.N.O (2011): A Continuous Formulation of A( $\square$ )-Stable Second Derivative Linear Multistep Methods for Stiff IVPs in ODEs. Journal of Algorithms and Computational Technology, Vol. 6, No. 1: 79-100

[27]. Owolabi, K.M. (2013): An Efficient Implicit Optimal Order Formula for Direct Integration of Second Order Orbital Problems. International Journal of Nonlinear Science, Vol. 16, No. 2: 175-184

[28]. Ra'ft Abdelrahim and Zurni Omar (2016): One-Step Hybrid Block Method with One Generalized Off-Step Points for Direct Solution of Second Order Ordinary Differential Equations. Applied Mathematical Sciences, Vol. 10, No. 29: $1423-1432$

[29]. Sagir, A.M (2014): Numerical Treatment of Block Method for the Solution of Ordinary Differential Equations. International Journal of Mathematical, Computational, Physical, Electrical and Computer Engineering, Vol. 8, No. 2: $259-263$

[30]. Sahi, R.K., Jator, S.N., and Khan, N.A. (2012): A Simpson's-Type Second Derivative Method for Stiff Systems. International Journal of Pure and Applied Mathematics, Vol. 81, No. 4: 619-633 